Results Between 2010-2018, 5681 syphilis cases were diagnosed in BC, including $61(1.1 \%)$ diagnosed with OS. The proportion of syphilis cases with OS increased from $0.48 \%$ in 2010 to $2.99 \%$ in 2018 ( $\mathrm{P} \leq 0.05)$. Among OS cases, median age was 47 years, $88.5 \%$ were male and the most common ophthalmologic diagnosis was panuveitis (44.3\%). At the time of syphilis diagnosis, $50.8 \%$ of OS cases were HIV-positive compared to $25.8 \%$ of controls $(\mathrm{P} \leq 0.001)$. Among individuals with HIV co-infection, OS cases had higher viral loads (42.9\% vs $79.7 \%$ were suppressed; $\mathrm{P} \leq 0.001)$ and lower median CD4 counts (470 vs 615 cells $/ \mathrm{mm}^{3} ; \mathrm{P} \leq 0.05$ ) than controls. Factors associated with OS were primary/secondary stage of syphilis (adjusted odds ratio [aOR] 4.06; 95\% confidence interval $[\mathrm{CI}]$ 1.52-10.8), early latent stage of syphilis (aOR 3.71; 95\%CI 1.39-9.95), and HIV-positive serostatus (aOR $2.49 ; 95 \%$ CI $1.27-4.88)$.

Conclusion OS incidence and proportion increased over the study period in BC. These findings highlight the importance of vigilance for OS, particularly those in the early stages of syphilis and those living with HIV, to avoid diagnostic and treatment delays.

Disclosure No significant relationships.

\section{P124 ATTITUDES AND BELIEFS REGARDING HIV AND AIDS AMONG IMMIGRANTS IN MOPANI DISTRICT, SOUTH AFRICA}

Hilda Shilubane* „_unic Khoza. University of Venda, Thohoyandou, South Africa

\subsection{6/sextrans-2019-sti.301}

Background Sub-Saharan Africa faces and is severely affected by many conflicts. Human Immunodeficiency Virus (HIV) and Acquired Immunodeficiency Syndrome (AIDS) threaten both the physical and financial well-being of individuals in struggling countries. Research indicates that negative attitude towards HIV/AIDS as well as persons infected with the virus persist despite the increased knowledge of prevention. This research aims to explore and describe the immigrants' attitudes and beliefs regarding HIV and AIDS in the Mopani district, Limpopo Province.

Methods A quantitative descriptive design was used. The target population was all immigrants at Mopani district in Limpopo province and the sample comprised of males and females. Data was collected using a questionnaire with closed and open-ended questions. Ethical standards were maintained.

Results The study revealed that many participants expressed discriminatory practice towards individuals infected by HIV and those with AIDS. Many viewed promiscuity and the disease called Makhume (meaning illness caused by the omission of purification rites following the death of a family member) as the cause for HIV and AIDS.

Conclusion The findings may hinder the achievement of healthy lives and promotion of well-being at all ages if not addressed appropriately. The collaboration of various departments (Health, Social development, Education, and etc.) in the Mopani district is required to change these negative attitudes and beliefs that influence immigrants' behaviour. In addition, the use of audio-visuals and peer teaching may help to change their attitudes since they have shown to be most successful in changing the attitudes.

Disclosure No significant relationships.

\section{P125 LOW RATE OF HIV TESTING AMONG PRISONERS IN IRAN: FINDINGS FROM NATIONWIDE REPEATED BEHAVIORAL SURVEYS}

${ }^{1}$ Mohammad Karamouzian*, ${ }^{2}$ Najmeh Parhizgari, ${ }^{3}$ Mostafa Shokoohi, ${ }^{3}$ Fatemeh Tavakoli, ${ }^{3}$ Armita Shahesmaeili, ${ }^{4}$ Ali Mirzazadeh, ${ }^{3}$ Hamid Sharifi. ${ }^{1} B C$ Center on Substance Use, Vancouver, Canada; ${ }^{2}$ Tehran University of Medical Sciences, Tehran, Iran; ${ }^{3}$ WHO Collaborating Center for HIVISTI Surveillance, KERMAN, Iran; ${ }^{4}$ UCSF, San Francisco, USA

\subsection{6/sextrans-2019-sti.302}

Background Prisoners are disproportionately at increased risk of HIV infection. Despite the importance of assessing HIV testing rates among key populations at risk of HIV, our understanding of HIV testing practices of Iranian prisoners is limited. This study utilizes data from two repeated behavioral survey studies to shed light on the prevalence and correlates of HIV testing among Iranian prisoners.

Methods Data were obtained from two consecutive surveys in $2009(\mathrm{~N}=5913)$ and $2013(\mathrm{~N}=5430)$ using a multistage random sampling approach including both large and small prison settings. Having a recent HIV test result was defined as having an HIV test result over the last 12 months. Biological and survey data were collected through dried blood spot testing and standardized risk assessment questionnaire, respectively. Descriptive statistics including frequencies, percentages, and $95 \%$ confidence intervals (CI) were reported. Bivariable and multivariable logistic regression models were built to compare the odds of having a recent HIV test result among different subgroups of prisoners.

Results Most participants had never tested for HIV $(68.7 \%$ in 2009 and $62.2 \%$ in 2013). Only 9.9\% (in 2009) and $11.6 \%$ (in 2013) of the participants had a recent HIV test result. Older age $(\mathrm{AOR}=1.50)$, lifetime history of drug use $(\mathrm{AOR}=1.87)$ and sexual intercourse $(\mathrm{AOR}=1.42)$, and self-perceived risk of $\mathrm{HIV}(\mathrm{AOR}=0.74)$ were significantly associated with recent HIV testing in 2009. Female sex $(\mathrm{AOR}=4.03)$, multiple incarcerations $(\mathrm{AOR}=1.40)$, alcohol consumption $(\mathrm{AOR}=1.23)$, and STI $(\mathrm{AOR}=1.79)$ and HIV knowledge $(\mathrm{AOR}=1.62)$ were significant correlates in 2013 .

Conclusion HIV testing is extremely low among prisoners in Iran. There is an urgent need to revisit current HIV testing policies across Iranian prisons and identify the barriers to HIV testing within the prison settings. Scaling up rapid tests as well as routine opt-out HIV testing services could help encourage prisoners to further use the available harm reduction facilities inside prisons.

Disclosure No significant relationships.

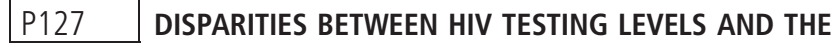 SELF-REPORTED HIV-NEGATIVE STATUS OF SEXUALLY ACTIVE COLLEGE STUDENTS}

Edmond Pui Hang Choi ${ }^{1 *}$, Janet Yuen Ha Wong ${ }^{2}$, Daniel Yee Tak Fong ${ }^{1}{ }^{1}$ The University of Hong Kong, School of Nursing, Hong Kong, PRC; ${ }^{2}$ The University of Hong Kong, PRC

\subsection{6/sextrans-2019-sti.303}

Background Despite the availability of rapid point-of-care human immunodeficiency virus (HIV) testing, the use of a self-reported HIV status has its own value, especially in research studies. Sexually active people without prior HIV testing should report as 'HIV status unknown' or 'never tested' instead of 'HIV negative' when they answer the question item on HIV status. The study aimed to assess the 